\title{
Mochizuki's Crys-Stable Bundles: A Lexicon and Applications
}

\author{
By \\ Brian OSSERMAN*
}

\begin{abstract}
Mochizuki's work on torally crys-stable bundles [18] has extensive implications for the theory of logarithmic connections on vector bundles of rank 2 on curves, once the language is translated appropriately. We describe how to carry out this translation, and give two classes of applications: first, one can conclude almost immediately certain results classifying Frobenius-unstable vector bundles on curves; and second, it follows from the results of [22] that one also obtains results on rational functions with prescribed ramification in positive characteristic.
\end{abstract}

\section{$\S 1 . \quad$ Introduction}

Mochizuki's theory of torally crys-stable bundles and torally indigenous bundles developed in [18] has, after appropriate translation, immediate implications for logarithmic connections on vector bundles of rank 2 on curves. This in turn has immediate implications to a subject which has recently been studied by a number of different people (see, for instance, [15], [14], [8], [20]): Frobeniusunstable vector bundles, and by extension the generalized Verschiebung rational map induced on moduli spaces of vector bundles by pulling back under Frobenius. Furthermore, together with the results of [22], one can use Mochizuki's work to describe rational functions with prescribed ramification in positive characteristic. This relationship provided the original motivation for the ultimately

Communicated by S. Mochizuki. Received October 14, 2004. Revised April 5, 2005, September 8, 2005.

2000 Mathematics Subject Classification(s): 14H60.

This paper was supported by a fellowship from the Japan Society for the Promotion of Science.

* Department of Mathematics, University of California, Berkeley, CA 94720-3840, USA.

e-mail: osserman@math.berkeley.edu 
self-contained arguments of [23], but also goes further by giving a finiteness result not yet known by direct arguments.

However, because the theory of [18] was aimed towards unrelated $p$-adic applications, it does not contain any translation of its results back into the language of vector bundles with connection or Frobenius-unstable vector bundles, and until recently remained outside the circle of literature treating these topics. The aim of this paper is therefore primarily expository: we will give a lexicon of Mochizuki's language and a survey of certain key results, and then detail the applications described above. The arguments in Section 3 were suggested almost uniformly by Mochizuki, but any blame for the particular translation in Section 2 of Mochizuki's work into the language of vector bundles lies solely with the author.

\section{$\S 2 . \quad$ The Lexicon}

Fix $g, r \geq 0$ with $2 g-2+r>0$, and $p$ an odd prime. Let $S=\operatorname{Spec} A$ with $A$ local and defined over its residue field $k$. Now, let $C$ be a smooth proper curve of genus $g$, with $r$ disjoint sections $P_{1}, \ldots, P_{r}$. For technical reasons, we allow $A$ to be any Noetherian, strictly Henselian local ring of characteristic $p$. However, for conceptual purposes, it suffices to take $S=\operatorname{Spec} k$, for an algebraically closed field $k$ of characteristic $p$. In this section, we give a series of statements expressing definitions and results of [18] in terms of connections on vector bundles; the proofs are deferred until the next section. We begin by setting terminological conventions for some standard concepts.

Although it will not arise until later, we denote by $C^{(p)}$ the $p$-twist of $C$ over $S$, and Frobenius will always refer to the relative Frobenius map $F: C \rightarrow C^{(p)}$.

Definition 2.1. Given a vector bundle $\mathscr{E}$ on $C$, a logarithmic $S$ connection on $\mathscr{E}$ is an $\mathcal{O}_{S}$-linear map $\nabla: \mathscr{E} \rightarrow \Omega_{C / S}^{1}\left(\sum_{i}\left[P_{i}\right]\right) \otimes \mathscr{E}$, satisfying the connection rule $\nabla(f s)=f \nabla(s)+d f \otimes s$. For any $i$, a logarithmic $S$-connection has a canonical residue $\operatorname{Res}_{P_{i}} \nabla \in \operatorname{End}\left(\left.\mathscr{E}\right|_{x}\right)$ induced by the residue map $\Omega_{C / S}^{1}\left(P_{i}\right) \rightarrow \Gamma\left(S, \mathcal{O}_{S}\right)$. We will sometimes say regular connection to emphasize that a connection is not logarithmic: i.e., that it takes values in $\Omega_{C / S}^{1} \otimes \mathscr{E}$.

Definition 2.2. $\quad$ Given a vector bundle $\mathscr{E}$ on $C$, a sub-bundle $\mathscr{F}$ of $\mathscr{E}$ is said to be destabilizing (respectively, weakly destabilizing) if the slope of $\mathscr{F}$ is strictly greater than (resp., greater than or equal to) the slope of $\mathscr{E}$. We denote by $\operatorname{End}^{0}(\mathscr{E}) \subset \operatorname{End}(\mathscr{E})$ the sheaf of endomorphisms with vanishing trace. 
Because $S$ has characteristic $p$, there is a notion of $p$-curvature associated to $\nabla$ (see $[12, \S 5]$ ), which allows us to define:

Definition 2.3. We say that a logarithmic $S$-connection $\nabla$ has $p$ trivial determinant if the determinant connection $\operatorname{det} \nabla$ on $\operatorname{det} \mathscr{E}$ is a regular connection with vanishing $p$-curvature.

As the name suggests, this definition is intended to generalize trivial determinant to cases where the stricter notion wouldn't make sense - for instance, when $\mathscr{E}$ has determinant of degree $p$. We also remark that the regularity of the determinant connection implies that the residues of $\nabla$ have vanishing trace.

For each $i$, fix a $\rho_{i} \in \Gamma\left(S, \mathcal{O}_{S}\right) /\{ \pm 1\}$. The fundamental object we will deal with is, in Mochizuki's language, the torally crys-stable bundle (see [18, Def. I.1.2, p. 89]). In our lexicon, we have the following equivalence:

Proposition 2.4. A torally crys-stable bundle on $C / S$ of radii $\left\{\rho_{i}\right\}$ is equivalent to an equivalence class of vector bundles $\mathscr{E}$ of rank 2 on $C$, together with logarithmic $S$-connection $\nabla$ having p-trivial determinant, satisfying the following conditions:

(i) for each $i, \operatorname{Tr}\left(\left(\operatorname{Res}_{P_{i}} \nabla\right)^{2}\right)=2 \rho_{i}^{2}$;

(ii) at the closed point of $S, \operatorname{Res}_{P_{i}} \nabla$ is non-zero for all $i$;

(iii) for every geometric point of $S$, after restriction to that point there is no weakly destabilizing sub-bundle of $\operatorname{End}^{0}(\mathscr{E})$ which is horizontal with respect to the connection induced by $\nabla$.

The equivalence relation is that obtained by allowing tensoring by any line bundle with S-connection; note that conditions (i)-(iii) are invariant under such tensoring.

Remark 2.5. We note the following:

(i) Assuming (as always) that all ranks are prime to $p$, two connections with $p$-trivial determinant on two bundles may differ by tensoring by a line bundle with connection only if the latter has trivial $p$-curvature: indeed, this follows the fact that the $p$-curvature of the tensor product connection is essentially the sum of the $p$-curvatures of the connections, which can be seen from the functorial definition of $p$-curvature given below, or via explicit calculations from the standard definition as in [20, Cor. 3.6 (iii)]. 
(ii) Since $\rho_{i}$ was only chosen up to sign, in the case that $S$ is integral we see that the radii of a torally crys-stable bundle are determined by $\nabla$, and do not constitute additional data.

(iii) If for any $i, \operatorname{Res}_{P_{i}} \nabla$ is diagonalizable, condition (i) will be satisfied for $\rho_{i}$ equal to the eigenvalues of the corresponding residue (which will necessarily be paired with opposite sign).

(iv) Although condition (iii) is very closely related to the non-existence of a horizontal destabilizing sub-bundle of $\mathscr{E}$, describing the relationship precisely is complicated, and as stated below, will not be necessary in the case of positive level, which will be our main focus.

We now give equivalent definitions of certain other terms of Mochizuki's theory which will be relevant. First, we define:

Definition 2.6. Let $(\mathscr{E}, \nabla)$ be a logarithmic $S$-connection on $C$, where $\mathscr{E}$ is of rank 2, and $\mathscr{L}$ a line sub-bundle of $\mathscr{E}$. Then the Kodaira-Spencer map

$$
\kappa: \mathscr{L} \rightarrow \mathscr{E} / \mathscr{L} \otimes \Omega_{C / S}^{1}\left(\sum_{i}\left[P_{i}\right]\right)
$$

associated to $(\mathscr{E}, \nabla)$ and $\mathscr{L}$ is the $\mathcal{O}_{C}$-linear map obtained by applying $\nabla$ to the natural inclusion and quotient maps. When $\mathscr{E}$ is unstable, we will refer to the Kodaira-Spencer map of $(\mathscr{E}, \nabla)$ to mean the Kodaira-Spencer map associated to $(\mathscr{E}, \nabla)$ and the destabilizing sub-bundle of $\mathscr{E}$ (see Lemma 3.4 below).

Note that the condition that a line sub-bundle $\mathscr{L}$ be horizontal for $\nabla$ is equivalent to the condition that its Kodaira-Spencer map vanish.

Proposition 2.7. $\quad$ Let $(\mathscr{E}, \nabla)$ be a representative of a torally crys-stable bundle. Then $(\mathscr{E}, \nabla)$ has level $\ell$ if and only if:

(i) in the case $\ell=0$, the restriction of $\mathscr{E}$ to every geometric point of $S$ is semistable;

(ii) in the case $\ell>0, \mathscr{E}$ has a line sub-bundle $\mathscr{L}$ of degree $\ell+\frac{1}{2} \operatorname{deg} \mathscr{E}$ such that the Kodaira-Spencer map associated to $(\mathscr{E}, \nabla)$ is generically nonzero on every fiber of $C \rightarrow S$, and is an isomorphism at the $P_{i}$.

Furthermore, any bundle with connection satisfying all conditions for crysstability of Proposition 2.4 except possibly condition (iii), and also satisfying the present condition (ii), is necessarily crys-stable. 
In fact, one checks that because of condition (iii) of Proposition 2.4, in the case that there are no marked points, a destabilizing line sub-bundle necessarily satisfies condition (ii) of Proposition 2.7, so the level of a torally crys-stable bundle is independent of $\nabla$, and simply measures the failure of $\mathscr{E}$ to be semistable. Note however that unless $S=\operatorname{Spec} k$, it is not necessarily the case that any torally crys-stable bundle has a level.

It immediately follows (see [18, Lem. I.3.4, p. 104]) that we can define:

Definition 2.8. A torally indigenous bundle is a torally crys-stable bundle of level $\frac{1}{2}(2 g-2+r)$.

Note that because of degree considerations, being a torally indigenous bundle is equivalent to $\kappa$ being an isomorphism.

Finally, we can state:

Proposition 2.9. A torally crys-stable bundle represented by $(\mathscr{E}, \nabla)$ is dormant if and only if the p-curvature of $\nabla$ is zero. The radii of a dormant torally crys-stable bundle are necessarily in $\mathbb{F}_{p} /\{ \pm 1\}$.

Note that here we use the hypothesis of Proposition 2.4 that our connections have $p$-trivial determinant, as we otherwise would not have that vanishing $p$-curvature is preserved under equivalence.

The results of Mochizuki central for our purposes can now be stated:

For the following two theorems, we fix radii $\left\{\rho_{i}\right\}$ lying in $\mathbb{F}_{p} /\{ \pm 1\}$.

Theorem 2.10 (Mochizuki). The stack of dormant torally indigenous bundles of radii $\left\{\rho_{i}\right\}$ is finite flat over $\overline{\mathcal{M}}_{g, r}$, and étale over points corresponding to totally degenerate curves.

Theorem 2.11 (Mochizuki). Suppose that $p \geq 2 g+r-2 \ell$, and $\ell>0$. Then the stack of dormant torally crys-stable bundles of radii $\left\{\rho_{i}\right\}$ and level $\ell$ is flat over $\overline{\mathcal{M}}_{g, r}$ of relative dimension $2 g-2+r-2 \ell$.

\section{$\S 3 . \quad$ Proofs}

The purpose of this section is to prove the statements of the preceding section, as well as certain lemmas which will be of use for our applications. We begin with some remarks on projective bundles and connections which hold for vector bundles of arbitrary rank $n$, but we assume throughout that $p$ does not divide $n$. 
First, given a projective bundle $\mathscr{P}$ on a relative smooth, proper curve $C$ over an arbitrary Noetherian scheme $S$, it is well-known that étale locally on $S$, we may write $\mathscr{P}=\mathbb{P}(\mathscr{E})$ for some vector bundle $\mathscr{E}$ on $C$. To see this, we use that $\mathscr{P}=\mathbb{P}(\mathscr{E})$ if and only if there exists a line bundle on $\mathscr{P}$ which is $\mathcal{O}(1)$ on fibers, from which one can obtain $\mathscr{E}$ via push-forward. First, by Tsen's theorem one can remove the Brauer obstruction for such an $\mathscr{E}$ on any special fiber after étale base change (see, e.g., [6, Cor. 5.8, p. 132]). Next, one can extend to the formal completion on the base by vanishing of obstructions to deforming the $\mathcal{O}(1)$ : indeed, if $\mathscr{P}_{0}$ and $C_{0}$ denote restrictions to the closed fiber, these obstructions lie in $H^{2}\left(\mathscr{P}_{0}, \mathcal{O}_{\mathscr{P}_{0}}\right)$, which one can check vanishes via the Leray spectral sequence obtained by pushing forward $\mathcal{O}_{\mathscr{P}_{0}}$ to $C_{0}$. One can effectivize this deformation (i.e., extend to the complete local ring on the base) by [24, Cor. 5.1.6]. Finally, one can apply the Artin approximation theorem [2, Cor. $2.2]$ to construct $\mathscr{E}$ after a further étale base change, using [25, Prop. 8.9.1] to reduce to the case that $S$ is of finite type over Spec $\mathbb{Z}$.

We now return to the hypothesis that $S$ is strictly Henselian. Given the above construction, since $\mathscr{E}$ is unique up to tensoring by line bundles, and by the invariance of degree in families, we may define:

Definition 3.1. $\quad$ Let $\mathscr{P}$ be a $\mathbb{P}^{n-1}$-bundle on a smooth relative curve $C / S$. Then the degree class of $\mathscr{P}$ is defined to be the congruence class modulo $n$ of the degree of any $\mathscr{E}$ on $C$ with $\mathscr{P} \cong \mathbb{P}(\mathscr{E})$.

Note that the degree class could be defined more intrinsically as the class induced by $\mathscr{P}$ in $H_{\text {ét }}^{2}\left(C, \mu_{n}\right)$; however, the more elementary definition will also be more immediately useful.

There are a number of issues involving translation between the formalities of the Grothendieck point of view on connections on sheaves and the classical definitions, and similarly for the formalism of log structures. However, such translations are well-known, and we refer the reader to [4, Prop. 2.9], [11], and [19, p. 13]. To go between connections on $\mathscr{P}$ and connections on some $\mathscr{E}$ with $\mathbb{P}(\mathscr{E})=\mathscr{P}$, one could extend the above argument for producing such an $\mathscr{E}$ by working with an $\mathcal{O}(1)$ obtained as an $n$th root of the relative canonical bundle of $\mathscr{P}$ over $C$. However, we will take a somewhat more naive approach.

Definition 3.2. Let $\mathscr{E}$ be a vector bundle on $C / S$. Then an $S$ connection $\nabla$ on $\mathbb{P}(\mathscr{E})$ is a global section of the sheaf PConn obtained as the sheafification of the presheaf whose sections over any $U \subset C$ are equivalence classes of $S$-connections on $\left.\mathscr{E}\right|_{U}$, defined up to tensoring by connections on $\mathcal{O}_{U}$. If $C$ has sections $P_{1}, \ldots, P_{r}$, a logarithmic $S$-connection $\nabla$ on $\mathbb{P}(\mathscr{E})$ is a global 
section of PConn ${ }^{\log }$, defined as above, but with logarithmic $S$-connections instead of regular ones.

Such a connection on $\mathscr{E}$ is easily checked to yield a connection in the Grothendieck sense on $\mathbb{P}(\mathscr{E})$ as a scheme over $C$, so we have a map from the sheaf PConn on $C$ to the sheaf of connections in the Grothendieck sense on $\mathbb{P}(\mathscr{E})$ on $C$. Now, because $p$ is prime to $n$, both sheaves are pseudo-torsors over $\operatorname{End}^{0}(\mathscr{E}) \otimes \Omega_{C / S}^{1}$ : in the case of PConn, any two connections on $\mathscr{E}$ differ naturally by an element of $\operatorname{End}(\mathscr{E}) \otimes \Omega_{C / S}^{1}$, and modding out by tensoring with connections on line bundles yields the quotient sheaf $\operatorname{End}^{0}(\mathscr{E}) \otimes \Omega_{C / S}^{1}$; in the case of connections in the Grothendieck sense on $\mathbb{P}(\mathscr{E})$, we simply use the fact that $\operatorname{End}^{0}(\mathscr{E})$ is the sheaf of infinitesimal automorphisms of $\mathbb{P}(\mathscr{E})$. Because $\mathscr{E}$ is locally trivial, so has connections locally, we find that in fact both sheaves are torsors, and not merely pseudo-torsors, and since the torsor structures are natural and compatible, we conclude that the sheaves are in fact isomorphic, and our naive definition of connection agrees with the Grothendieck definition. The same argument applies in the logarithmic case, with $\Omega_{C / S}^{1}$ replaced by $\Omega_{C / S}^{1}\left(\sum_{i}\left[P_{i}\right]\right)$, and using the appropriate formalism of log schemes to define logarithmic connections in the Grothendieck sense.

Proposition 3.3. $\quad$ Let $\left(\mathscr{P}, \nabla^{\mathscr{P}}\right)$ be a projective bundle of dimension $n-$ 1 with (possibly logarithmic) connection on $C / S$. Let $\mathscr{L}$ be a line bundle whose degree modulo $n$ is the degree class of $\mathscr{P}$, and with regular connection $\nabla^{0}$. Then there exists an $\mathscr{E}$ of determinant $\mathscr{L}$ on $C / S$ such that $\mathbb{P}(\mathscr{E}) \cong \mathscr{P}$, and for any such $\mathscr{E}$, there exists a unique connection $\nabla$, logarithmic if $\nabla^{\mathscr{P}}$ was, whose determinant is $\nabla^{0}$, and such that $\nabla$ recovers $\nabla^{\mathscr{P}}$ under projectivization.

Proof. Let $\mathscr{E}^{\prime}$ be any vector bundle such that $\mathbb{P}\left(\mathscr{E}^{\prime}\right) \cong \mathscr{P}$. Since $\mathscr{L}$ is of the appropriate degree class, and multiplication by $n$ is étale on the Jacobian, we find that there exists an $\mathscr{L}^{\prime}$ with $\mathscr{L}^{\prime \otimes n} \cong \mathscr{L} \otimes\left(\operatorname{det} \mathscr{E}^{\prime}\right)^{-1}$, and setting $\mathscr{E}=\mathscr{E}^{\prime} \otimes \mathscr{L}^{\prime}$ will give us $\operatorname{det} \mathscr{E} \cong \mathscr{L}$. Next, since $p$ does not divide $n, \nabla$ will certainly be uniquely determined by its determinant and projectivization, if it exists. Moreover, everything is compatible with restriction, so by uniqueness it suffices to produce $\nabla$ on an open cover of $C$, since the restrictions on overlaps will then have to glue. Let $U$ be an open set on which $\nabla^{\mathscr{P}}$ is represented by some $\nabla^{U}$ on $\left.\mathscr{E}\right|_{U}$. We then see that by tensoring with $\left(\mathcal{O}_{U}, \frac{1}{n}\left(\nabla^{0}-\operatorname{det} \nabla^{U}\right)\right)$, we obtain the desired $\nabla$ on $U$.

Specializing now to $n=2$, the first part of the following lemma is standard: 
Lemma 3.4. Let $C / S$ be a smooth, proper curve over a connected scheme $S$, and $\mathscr{E}$ a vector bundle of rank 2 on $C$. Suppose that $\mathscr{L} \subset \mathscr{E}$ is a weakly destabilizing line sub-bundle of $\mathscr{E}$. Then $\operatorname{deg} \mathscr{L}$ is uniquely determined, and if $\mathscr{L}$ is in fact destabilizing, $\mathscr{L}$ itself is uniquely determined.

Furthermore, in the case that $\mathscr{L}$ is destabilizing, if further we are given a (possibly logarithmic) connection $\nabla$ on $\mathscr{E}$, such that $\mathscr{L}$ is not horizontal for $\nabla$, it follows that $\operatorname{End}^{0}(\mathscr{E})$ has no weakly destabilizing sub-bundles horizontal for the connection $\nabla^{\prime}$ induced by $\nabla$ on $\operatorname{End}^{0}(\mathscr{E})$.

Proof. The only observation required for the first part is that even in the general relative setting, Nakayama's lemma gives the statement that a line bundle of negative degree has no non-zero global sections. The assertions then follow by standard arguments involving composing the inclusion of one line sub-bundle with the quotient map induced by another, and vice versa.

For the second part, we first note that if $\mathscr{L}^{0}$ denotes the line sub-bundle of $\operatorname{End}^{0}(\mathscr{E})$ sending $\mathscr{L}$ to $0, \mathscr{L}^{0} \cong \mathcal{H}$ om $(\mathscr{E} / \mathscr{L}, \mathscr{L})$ and thus has positive degree. Also, $\mathscr{L}^{0}$ is not horizontal for $\nabla^{\prime}$. However, the image of $\mathscr{L}^{0}$ under $\nabla^{\prime}$ is contained in $\mathscr{F} \otimes \omega$, where $\mathscr{F}$ denotes the sub-bundle of $\operatorname{End}^{0}(\mathscr{E})$ sending $\mathscr{L}$ to $\mathscr{L}$, and $\omega=\Omega_{C / S}^{1}$ in the case of a regular connection, or $\Omega_{C / S}^{1}\left(\sum_{i}\left[P_{i}\right]\right)$ in the logarithmic case. A local calculation shows that the image of an element of $\mathscr{F}$ under $\nabla^{\prime}$ is contained in $\mathscr{F} \otimes \omega$ if and only if the element is in fact in $\mathscr{L}^{0} \subset \mathscr{F}$. It now follows that no proper sub-bundle of $\operatorname{End}^{0}(\mathscr{E})$ containing $\mathscr{L}^{0}$ can be horizontal for $\nabla^{\prime}$ : indeed, such a sub-bundle would have to contain $\mathscr{F}$, hence strictly contain it, hence be all of $\operatorname{End}^{0}(\mathscr{E})$. Next, we observe that given a weakly destabilizing sub-bundle $\mathscr{F}^{\prime}$ of $\operatorname{End}^{0}(\mathscr{E})$ of rank 2 , its quotient $\mathscr{Q}$ would have non-positive degree, and hence the composed map $\mathscr{L}^{0} \rightarrow \mathscr{Q}$ is necessarily 0 ; but this implies that $\mathscr{F}^{\prime}$ contains $\mathscr{L}^{0}$, hence also $\mathscr{F}$, i.e., that $\mathscr{F}^{\prime}=\mathscr{F}$, hence is not horizontal for $\nabla$. Finally, the rank 1 case follows from the rank 2 case by the self-duality of $\operatorname{End}^{0}(\mathscr{E})$.

We now give the proofs of the main statements of the previous section.

Proof of Proposition 2.4. Given a vector bundle with connection $(\mathscr{E}, \nabla)$, we first want to check that conditions (i)-(iii) of the proposition are equivalent to conditions $(1)-(3)$ of $[18$, Def. 1.2, p. 89$]$ for the projectivization $\mathbb{P}(\mathscr{E})$ with the induced connection. For conditions (i) and (ii), one need only know that Mochizuki's monodromy operator $\mu_{i}$ is simply the residue of $\nabla$ at $P_{i}$. Then, because $\operatorname{Ad}(\mathbb{P}(\mathscr{E})) \cong \operatorname{End}^{0}(\mathscr{E})$, the equivalence for condition (iii) is immediate. Now, condition (4) of loc. cit. is vacuous since we have restricted to smooth curves, so we find that given a vector bundle with connection $(\mathscr{E}, \nabla)$ satisfying 
the condition of the proposition, we get a torally crys-stable bundle simply by projectivizing.

Conversely, we want to show that, since our base is strictly Henselian, given a torally crys-stable bundle $(\mathscr{P}, \nabla)$ we can find a vector bundle with connection having $p$-trivial determinant, and such that the projectivization recovers $(\mathscr{P}, \nabla)$. By Proposition 3.3 , we need only produce a line bundle $\mathscr{L}$ with the appropriate degree modulo $n$, and having a connection with vanishing $p$-curvature. But letting $\mathscr{L}^{\prime}$ be any line bundle on $C^{(p)}$ of degree congruent to $\frac{1}{p}$ times the desired degree modulo $n$, we can take $\mathscr{L}:=F^{*} \mathscr{L}^{\prime}$, and the induced canonical connection gives us the desired connection with vanishing $p$-curvature.

We next have:

Proof of Proposition 2.7. We wish to compare our criteria to [18, Def. I.3.2, p. 103]. In light of the translation provided by the above argument, and using the equivalence between line sub-bundles of a rank- 2 vector bundle and sections of its projectivization, one need only check two statements: first, that if $h_{\mathscr{L}}$ denotes the section of $\mathbb{P}(\mathscr{E})$ associated to a line sub-bundle $\mathscr{L}$ of $\mathscr{E}$, then $h_{\mathscr{L}}^{*} \omega_{\mathbb{P}(\mathscr{E}) / C} \cong \operatorname{Hom}(\mathscr{E} / \mathscr{L}, \mathscr{L})$; and second, that condition (ii) of the proposition is equivalent to condition (2) of loc. cit. together with condition (iii) of Proposition 2.4. For the first, we consider the dual statement, and note that $h_{\mathscr{L}}^{*} \tau_{\mathbb{P}(\mathscr{E}) / C}$ is the first-order deformation space of $h_{\mathscr{L}}$, which is naturally identified with $\operatorname{Hom}(\mathscr{L}, \mathscr{E} / \mathscr{L})$.

For the second, by Lemma 3.4, we see that the existence of a non-horizontal destabilizing $\mathscr{L}$ in condition (ii) of the present proposition precludes the existence of a horizontal weakly destabilizing sub-bundle of $\operatorname{End}^{0}(\mathscr{E})$. Conversely, condition (iii) of Proposition 2.4 implies that $\mathscr{L}$ cannot be horizontal, so we obtain the desired equivalence.

Before discussing our definition of $p$-curvature, we need to discuss the lifting of Grothendieck-perspective connections to higher-order PD-neighborhoods. This is completely standard in the case of classical connections on a sheaf of modules on $C / S$; see, e.g., [4, Thm. 4.8]. Now, suppose we have a connection $\nabla$ on some object $\mathscr{P}$ over $C$, which for simplicity we assume to be a separated scheme over $C$; that is, if $C^{[2]}$ denotes the first-order neighborhood of the diagonal in $C \times{ }_{S} C$ (which is the same with or without divided powers), $\nabla$ is an $\mathcal{O}_{C^{[2]} \text { - }}$ linear isomorphism $p_{1}^{*} \mathscr{P} \cong p_{2}^{*} \mathscr{P}$, which gives the identity when restricted to the diagonal. We wish to show that (since $C$ is a curve, so any connection is automatically integrable), we obtain a unique lifting to $n$ th-order neighborhoods 
of the diagonal in $C \times{ }_{S}^{\mathrm{PD}} C$ for any $n$. Let $U \rightarrow \mathscr{P}$ be any affine cover; in particular, $U$ is affine over $C$, so $\mathcal{O}_{U}$ is quasi-coherent over $C$, and the connection on $\mathscr{P}$ induces a classical connection on $\mathcal{O}_{U}$, which furthermore is compatible with the algebra structure on $\mathcal{O}_{U}$. Now, by the classical theory, we can lift this connection uniquely to $n$ th-order neighborhoods, with the lift given by the formal expression $e^{\left(\nabla_{\frac{d}{d t}}\right) \otimes(1 \otimes t-t \otimes 1)}$ for some local coordinate $t$ : that is to say, in the notation of $[4, \S 4]$ (i.e., with $\mathcal{D}^{n}(1)$ denoting the $n$th order PD neighborhood of the diagonal in $C \times{ }_{S}^{\mathrm{PD}} C$ ), and with $\mathscr{E}:=\mathcal{O}_{U}$, we take the map $\mathscr{E} \rightarrow \mathscr{E} \otimes \mathcal{D}^{n}(1)$ given by $s \mapsto \sum_{i=0}^{n}\left(\nabla_{\frac{d}{d t}}\right)^{i} s \otimes(1 \otimes t-t \otimes 1)^{[i]}$, and extend by scalars to obtained the desired $\mathcal{D}^{n}(1)$-linear isomorphism $\mathcal{D}^{n}(1) \otimes \mathscr{E} \rightarrow \mathscr{E} \otimes \mathcal{D}^{n}(1)$. One checks that the product rule for exponentials implies that this lift preserves the compatibility with the algebra structure. By uniqueness, this lifting necessarily agrees on restriction to intersections of open sets in the cover, so it follows that one obtains a lifting of the connection on $\mathscr{P}$ to $n$ th-order as well.

We are now ready to define $p$-curvature from the Grothendieck perspective. The idea is to consider the following picture:

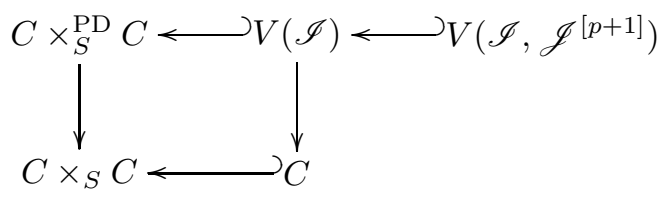

where the square is Cartesian, so that $\mathscr{I}$ is by definition the ideal in $C \times{ }_{S}^{\mathrm{PD}}$ $C$ generated by the ideal defining the diagonal in $C \times{ }_{S} C$. If we denote by $\mathscr{J}$ the ideal of the diagonal in $C \times{ }_{S}^{\mathrm{PD}} C$, we have $\mathscr{J}=\sum_{i \geq 1} \mathscr{I}^{[i]}$. Now, the main observation is that because $\left(\mathscr{I}, \mathscr{J}^{[p+1]}\right)=\left(\left\{\mathscr{I}^{[i]}\right\}_{i \neq 0, p}\right)$, one can check that $V\left(\mathscr{I}, \mathscr{J}^{[p+1]}\right)$ has structure sheaf isomorphic to $\mathcal{O}_{C} \oplus F^{*} \omega_{C / S}$, with multiplication determined by setting the product of any two elements of $F^{*} \omega_{C / S}$ to 0 . Given $\nabla$ an isomorphism between $p_{1}^{*} \mathscr{P}$ and $p_{2}^{*} \mathscr{P}$ on the firstorder neighborhood of the diagonal in $C \times{ }_{S} C$, we can pull back to $C \times{ }_{S}^{\mathrm{PD}}$ $C$, to obtain such an isomorphism on $V\left(\mathscr{J}^{[2]}\right)$, and we can then lift to $(p+$ 1)st order, obtaining an isomorphism on $V\left(\mathscr{J}^{[p+1]}\right)$, which we can restrict to $V\left(\mathscr{I}, \mathscr{J}^{[p+1]}\right)$. On the other hand, on $V(\mathscr{I})$, hence on $V\left(\mathscr{I}, \mathscr{J}{ }^{[p+1]}\right)$, we also have the trivial isomorphism between $p_{1}^{*} \mathscr{P}$ and $p_{2}^{*} \mathscr{P}$ obtained by factoring through the diagonal $C \subset C \times{ }_{S} C$; in particular, this is also the identity when restricted to the diagonal in $C \times{ }_{S}^{\mathrm{PD}} C$, so the difference of these two isomorphisms gives an automorphism of $p_{1}^{*} \mathscr{P}$ over $\operatorname{Spec} \mathcal{O}_{C} \oplus F^{*} \omega_{C / S}$, which is the identity modulo $F^{*} \omega_{C / S}$. Because of the square-zero structure on this sheaf, this corresponds to a section of $\operatorname{InfAut}(\mathscr{P}) \otimes F^{*} \omega_{C / S}$, where $\operatorname{InfAut}(\mathscr{P})$ is the sheaf of infinitesimal automorphisms of $\mathscr{P}$ over $C$. In the case of $\mathscr{P}=\mathbb{P}(\mathscr{E})$ 
for some vector bundle $\mathscr{E}$, we have $\operatorname{InfAut}(\mathscr{P})=\operatorname{End}^{0}(\mathscr{E})$. This section of $\operatorname{End}^{0}(\mathscr{E}) \otimes F^{*} \omega_{C / S}$ is then our $p$-curvature associated to $\nabla$.

We remark that these definitions can be checked to agree with those of [ 5 , A.1] when the latter is defined, which is when everything (including the base scheme) is smooth over a field.

Proof of Proposition 2.9. For the first assertion, we need only check the following two claims: first, that given any $\nabla$ on $\mathscr{E}$, the $p$-curvature of the induced connection on $\mathbb{P}(\mathscr{E})$ is simply the traceless part of the usual $p$-curvature of $\nabla$ on $\mathscr{E}$; and second, that a connection $\nabla$ on $\mathscr{E}$ with $p$-trivial determinant has $p$-curvature taking values in $\operatorname{End}^{0}(\mathscr{E}) \otimes F^{*} \omega_{C / S}$.

We begin by checking that the new definition of $p$-curvature agrees with the usual notion, when applied simply to $\mathscr{E}$. We can check this on local coordinates, so if we set $t$ to be a local coordinate for $C / S$, the derivations are locally generated by $\frac{d}{d t}$, and it suffices to compare definitions on this derivation, which satisfies $\left(\frac{d}{d t}\right)^{p}=0$. Thus, the usual definition of $p$-curvature is simply $\left(\nabla_{\frac{d}{d t}}\right)^{p}$. On the other hand, the lifting of our connection to the $(p+1)$ st order neighborhood in $C \times{ }_{S}^{\mathrm{PD}} C$ is given by the formula $\sum_{i=0}^{p}\left(\nabla_{\frac{d}{d t}}\right)^{i} \otimes(1 \otimes t-t \otimes 1)^{[i]}$, and when we mod out by $\mathscr{I}$, which is locally generated by $1 \otimes t-t \otimes t$, this kills all terms of positive order less than $p$, leaving only $1+\left(\nabla_{\frac{d}{d t}}\right)^{p} \otimes(1 \otimes t-t \otimes 1)^{[p]}$. The tautological map in the opposite direction is given simply by 1 , so our automorphism of $p_{1}^{*} \mathscr{E}$ is given simply by $1+(1 \otimes t-t \otimes 1)^{[p]} \otimes\left(\nabla_{\frac{d}{d t}}\right)^{p}$. But $(1 \otimes t-t \otimes 1)^{[p]}$ is precisely the square-zero element we used to obtain an infinitesimal automorphism of $\mathscr{E}$, so we find that the $p$-curvature from the new definition is also $\left(\nabla_{\frac{d}{d t}}\right)^{p}$, just as in the usual case.

One then easily checks our first claim: indeed, the functorial nature of the new definition of $p$-curvature implies that the section of $\operatorname{End}^{0}(\mathscr{E}) \otimes F^{*} \omega_{C / S}$ associated to the induced connection on $\mathbb{P}(\mathscr{E})$ is simply the traceless part of the $p$-curvature of $\nabla$ on $\mathscr{E}$, since this is precisely the description of the natural map $\operatorname{End}(\mathscr{E}) \cong \operatorname{InfAut}(\mathscr{E}) \rightarrow \operatorname{InfAut}(\mathbb{P}(\mathscr{E})) \cong \operatorname{End}^{0}(\mathscr{E})$. The second claim follows similarly, since the natural map $\operatorname{End}(\mathscr{E}) \cong \operatorname{InfAut}(\mathscr{E}) \rightarrow \operatorname{InfAut}(\operatorname{det} \mathscr{E}) \cong \mathcal{O}_{C}$ is precisely the trace map.

Finally, the assertion that the radii of a dormant torally crys-stable bundle lie in $\mathbb{F}_{p} /\{ \pm 1\}$ is the $n=0$ case of [18, Prop. II.1.5, p. 128].

We now address our statements of Mochizuki's results.

Proof of Theorem 2.10. This is the $n=0$ case of [18, Thm. II.2.8, p. $153]$. 
Proof of Theorem 2.11. We claim that it is enough to see that the stack of dormant torally crys-stable bundles of level $\ell$ is smooth over $\mathbb{F}_{p}$ of relative dimension $5 g-5+2 r-2 \ell$. Indeed, by standard commutative algebra it then suffices to show that the fibers of the map to $\overline{\mathcal{M}}_{g, r}$ have dimension at most $2 g-2+r-2 \ell$, which follows from [18, Thm. II.1.9, p. 132], after noting that the condition of dormancy gives a closed sub-functor of the functor determined by the condition of nilpotency (defined to be square nilpotency of the $p$-curvature of the connection), and that the condition on the characteristic insures that $\left(\overline{\mathcal{D}}_{g, r}^{\ell}\right)^{\prime}$ is in fact all of $\overline{\mathcal{D}}_{g, r}^{\ell}$. Note also the relevant statements on the map $\overline{\mathcal{D}}_{g, r}^{\ell} \rightarrow \overline{\mathcal{M}}_{g, r}$ following [18, Def. I.3.7, p. 105].

We have thus reduced down to the smoothness assertion. Over $\mathcal{M}_{g, r}$, this is the $j=0, i=\ell$ case of [18, Cor. III.1.6, p. 176], but we will need the full statement, whose proof is more involved, and generally follows the $n=0$ case of [18, Thm. II.2.8, p. 153]. As in loc. cit. (see in particular the remark on deformation theory and $\log$ schemes preceding the proof), we will check that our stack is log smooth, and smoothness in the (2-)category of stacks will follow formally. For the definition and properties of log deformations, see [10], and in particular Def. 8.1, Prop. 8.6, and Ex. 10.2. We take our base scheme $S$ to be $\operatorname{Spec} A$, where $A$ is an Artin ring which contains its separably closed residue field $k$, and suppose that we have $\left(C^{\log }, \mathscr{E}, \mathscr{L}, \nabla\right)$ a dormant torally crys-stable bundle of level $\ell$ on $C^{\log }$ over $S^{\log }$, where the $\log$ structure on $S$ is induced by pulling back from $\overline{\mathcal{M}}_{g, r}$, and on $C$ is the standard one, see $[18$, $\S$ I.1.1, p. 88]. We denote by a subscript 0 the restriction of these objects over $S$ to Spec $k$. We then take any exact closed immersion of log schemes $S^{\log } \rightarrow S_{1}^{\log }$ with underlying scheme $S_{1}=$ Spec $A_{1}$ being a small extension of $S$ over $\operatorname{Spec} k$, in the sense that $\operatorname{ker}\left(A_{1} \rightarrow A\right)$ is a square-zero principal $k$-module, hence in particular isomorphic to $k$. We therefore want to show that we can lift $\left(C^{\log }, \mathscr{E}, \mathscr{L}, \nabla\right)$ to $S_{1}^{\log }$, preserving the vanishing of the $p$-curvature. To do so, we will generally consider log deformations of the objects involved from $S^{\log }$ to $S_{1}^{\log }$, and the key is to consider the following maps

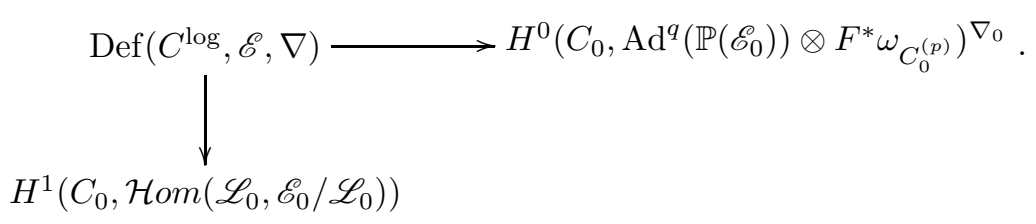

Here $\omega$ denotes the logarithmic differentials, and $\operatorname{Ad}^{q}\left(\mathbb{P}\left(\mathscr{E}_{0}\right)\right)$ denotes a certain subsheaf of $\operatorname{End}^{0}\left(\mathscr{E}_{0}\right)$ which is equal to the latter away from the marked points (see [18, p. 148]). Finally, $\operatorname{Def}\left(C^{\log }, \mathscr{E}, \nabla\right)$ denotes the space of logarithmic 
deformations of the triple from $S^{\log }$ to $S_{1}^{\log }$ : for $C^{\log }$, this is equivalent to classical deformations of $C$ which induce fixed deformations formally locally at each node (determined by the map of $\log$ structures of $S^{\log } \rightarrow S_{1}^{\log }$ ), together with deformations of each marked point and some additional data associated to the $\log$ structures at the nodes. The horizontal arrow is the $p$-curvature map, while the vertical arrow is the map giving the obstruction to deforming $\mathscr{L}$ inside the given deformation of $\mathscr{E}$; both will be examined in more detail shortly. We remark that we consider deformations up to tensoring with any line bundle with connection on any $C_{1}$ over $S_{1}$ which is trivial when restricted to $C$. We also denote by $\operatorname{Def}\left(C^{\log }\right)$ and $\operatorname{Def}{ }_{C_{1}^{\log }}(\mathscr{E}, \nabla)$ the spaces of logarithmic deformations of $C^{\log }$ over $S_{1}^{\text {log }}$, and of $(\mathscr{E}, \nabla)$ over a fixed deformation $C_{1}^{\text {log }}$ of $C^{\log }$, respectively.

The main point is that the triple $\left(C_{0}^{\log }, \mathscr{E}_{0}, \nabla_{0}\right)$ determines a locally free crystal in the logarithmic crystalline site over $S_{1}^{\text {log }}$, so if we have a logarithmic deformation $C_{1}^{\log }$ of $C^{\log }$, by considering it as a logarithmic PD-thickening of $C$, the theory of crystals gives a natural identification between the spaces

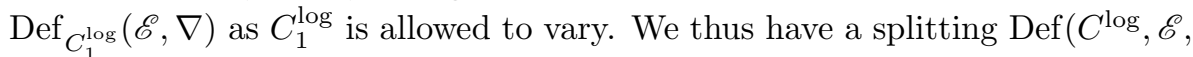
$\nabla)=\operatorname{Def}\left(C^{\text {log }}\right) \times \operatorname{Def}(\mathscr{E}, \nabla)$, where $\operatorname{Def}(\mathscr{E}, \nabla)$ is an abstract torsor over $H^{1}\left(C_{0}\right.$, $\left.\operatorname{End}^{0}\left(\mathscr{E}_{0}\right) \stackrel{\nabla_{0}}{\rightarrow} \operatorname{End}^{0}\left(\mathscr{E}_{0}\right) \otimes \omega_{C_{0}}\right)$. Moreover, under this splitting, the $p$-curvature map factors through $\operatorname{Def}(\mathscr{E}, \nabla)$. Now, the existence of the map measuring the obstruction to deforming $\mathscr{L}$ inside the deformation of $\mathscr{E}$ is addressed implicitly by the first isomorphism of [18, Prop. 1.7, p. 94]; however, one can also understand the map quite directly, using the fact that deformations of $\mathscr{L}_{0}$ within $\mathscr{E}_{0}$ are parametrized by $\mathcal{H o m}\left(\mathscr{L}_{0}, \mathscr{E}_{0} / \mathscr{L}_{0}\right)$, and standard Cech cocycle arguments. One checks that for a fixed element of $\operatorname{Def}(\mathscr{E}, \nabla)$, as the element of $\operatorname{Def}\left(C^{\text {log }}\right)$ varies from a fixed choice by an element of $H^{1}\left(C_{0}, \tau\left(C_{0}\right)\right)$, the obstruction class varies by the corresponding image of the natural map $H^{1}\left(C_{0}, \tau_{C_{0}}\right) \rightarrow$ $H^{1}\left(C_{0}, \mathcal{H} \operatorname{om}\left(\mathscr{L}_{0}, \mathscr{E}_{0} / \mathscr{L}_{0}\right)\right)$ induced by the Kodaira-Spencer map of $\nabla_{0}$ after tensoring by $\tau_{C_{0}^{\log }} \otimes \mathscr{L}_{0}^{-1}$, where $\tau_{C_{0}^{\log }}$ denotes the logarithmic tangent sheaf. Surjectivity of the obstruction map then follows from the hypothesis that the Kodaira-Spencer map is non-zero, and hence has a torsion sheaf for its cokernel.

To complete the proof, it was pointed out to the author by Mochizuki that the arguments and results [18, pp. 150-152] do not in fact make use of the torally indigenous hypothesis, requiring only that the bundle be torally crysstable. We then find that the $p$-curvature map is surjective by $[18$, Lem. 2.7, p. 152]. This surjectivity, together with the surjectivity of the obstruction map and our observations about the behavior of the two maps under our splitting, imply that we can find a deformation of $\left(C^{\log }, \mathscr{E}, \nabla\right)$ to $S_{1}^{\log }$ which has vanishing 
$p$-curvature and no obstruction to deforming $\mathscr{L}$, so we conclude the desired smoothness by standard deformation theory arguments over small extensions. Lastly, the dimension is the dimension of the simultaneous kernel of the $p$ curvature and obstruction maps. Again as in the proof of [18, Thm. II.2.8, p. 153], the kernel of the $p$-curvature map on $\operatorname{Def}(\mathscr{E}, \nabla)$ has dimension $3 g-3+r$, and by the surjectivity of the obstruction map, we obtain the dimension of the total kernel by adding $\operatorname{dim} \operatorname{Def}\left(C^{\log }\right)-\operatorname{dim} H^{1}\left(C_{0}, \mathcal{H o m}\left(\mathscr{L}_{0}, \mathscr{E}_{0} / \mathscr{L}_{0}\right)\right)=$ $3 g-3+r-(g-1+2 \ell)=2 g-2+r-2 \ell$, giving a total of $5 g-5+2 r-2 \ell$, as desired.

We conclude with two results stated entirely in terms of Mochizuki's language (in particular, they are over singular curves), but which we will need for our applications.

Proposition 3.5. $\quad$ A dormant torally indigenous bundle of radii $\left\{\rho_{i}\right\}$ on a nodal curve $C$ over Spec $k$ is equivalent to a collection of dormant torally indigenous bundles on each connected component $\tilde{C}_{i}$ of the normalization of the curve, where points on the $\tilde{C}_{i}$ lying above nodes are designated marked points, having radii $\left\{\rho_{i}\right\}$ at the points lying above marked points of $C$, and arbitrary radii at points lying above nodes, subject to the restriction that the radii of the two points lying above any given node must agree.

Proof. This follows immediately from [18, §I.4.4, p. 118], taking into account the fact that dormancy, being a condition of vanishing of $p$-curvature, will not be affected by the gluing.

We remark that the following lemma, whose proof is relatively straightforward from the results of [22] and [23], would be far more complicated if one attempted to directly apply Mochizuki's technique of considering totally degenerate curves, because such curves have no torally crys-stable bundles of intermediate level. In particular, by considering instead irreducible rational nodal curves, we avoid the machinery of PTCS bundles [18, §II.1.6, p. 137].

Lemma 3.6. Let $C$ be a general irreducible rational nodal curve over Spec $k$ of arithmetic genus $g \geq 2$, with no marked points. Then for any $\ell$ with $0<\ell \leq g-1$, there exists a dormant torally crys-stable bundle of level $\ell$ on $C$.

Proof. We make use of [22], which generalizes the construction of [18, $\S I V .2 .1]$ to the case of arbitrarily many marked points, and non-maximal level. Let $\tilde{C}$ be the normalization of $C$, with $2 g$ marked points lying above the nodes. 
We first consider the case that $\ell$ is an integer. Let $f$ be any function $\tilde{C} \cong \mathbb{P}^{1} \rightarrow$ $\mathbb{P}^{1}$ of degree $g-\ell$, and unramified at the marked points. By [22, Thm. 6.7] (with, in the notation of loc. cit., $d=\ell, n=2 g, m=g, \delta=0$, and $\alpha_{i}=\frac{p-1}{2}$ and $\beta_{i}=0$ for all $i$ ), this gives a dormant torally crys-stable bundle of level $\ell$ on $\tilde{C}$ with radii $\frac{p-1}{2}$ at every marked point, and by the previous proposition we can glue to obtain a dormant torally crys-stable bundle of level $\ell$ on $C$, as desired.

The case that $\ell$ is a strictly half-integer is similar, but somewhat more complicated. If we choose an identification $\tilde{C} \cong \mathbb{P}^{1}$ such that $\infty$ is not a marked point, we would like $f$ to have degree $\frac{p}{2}+g-\ell$, and be ramified to order at least $p$ at $\infty$. Of course, this is only possible if $\frac{p}{2}+g-\ell \geq p$; if in fact $\frac{p}{2}+g-\ell>p$, we can choose a rational function $f_{1}$ of degree $g-\ell-\frac{p}{2}$ and unramified at the marked points, and with the additional hypotheses that $f_{1}(\infty)=0$ and $f_{1}$ is unramified at $\infty$. We then set $f=f_{1}+x^{p}$, and observing that ramification indices $<p$ away from $\infty$ remain unchanged, we obtain a function of the desired degree and ramification, and again apply [22, Thm. 6.7], with $d=\frac{p}{2}+\ell, n=2 g, m=g-1, \delta=1$, and $\alpha_{i}=\frac{p-1}{2}$ and $\beta_{i}=0$ for all $i$.

Finally, if $\frac{p}{2}+g-\ell \leq p$, we set $e=p-1-(2 g-2-2 \ell)$, and $e^{\prime}=\frac{p \pm 1}{2}$ an odd integer according to the value of $p \bmod 4$. We claim that because $C$ and hence the marked points $P_{i}$ on $\tilde{C}$ are general, we can find a function $f_{1}$ of degree $\frac{1}{2}(p \pm 1+(2 g-1)(p-2-(2 g-2-2 \ell)))$ ramified to order $e^{\prime}$ at $P_{1}$ and $P_{2 g}$, and $e$ at $\infty$ and the remaining $P_{i}$, and unramified elsewhere, and mapping $\infty$ to $\infty$. Indeed, the last requirement can be achieved for any $f_{1}$ by applying an appropriate linear fractional transformation. Momentarily considering $\infty$ to be $P_{2 g+1}$, the claim then follows from the second formula of Theorem 5.5 below (see also [23] for a self-contained proof), by noting that if all $e_{i}^{\prime}$ are chosen to be equal to $e^{\prime}$, the conditions for existence of the desired $f_{1}$ are satisfied. We then set $f=f_{1}+x^{p}$, obtaining a function with the same ramification at $P_{1}, \ldots, P_{2 g}$, ramification of order $p$ at infinity, and degree $\frac{1}{2}(3 p \pm 1-2+(2 g-3)(p-2-(2 g-2-2 \ell)))$. As before, we apply [22, Thm. 6.7], with $d=\frac{p}{2}+\ell, n=2 g, m=g-1, \delta=1, \beta_{i}=0$ for all $i, \alpha_{1}=\alpha_{2 g}=\frac{p \mp 1}{4}$ and $\alpha_{i}=g-1-\ell+\frac{1}{2}$ for the remaining $i \leq 2 g$. The only additional difference is that since the radii are no longer all the same, we are required to glue $P_{1}$ to $P_{2 g}$ in forming our nodal curve. 


\section{$\S 4$. Frobenius-Unstable Vector Bundles}

In this section, we apply Theorem 2.11 to the study of the locus of Frobenius-unstable vector bundles on a curve. In particular, we restrict further to the case that $r=0$. While it would be convenient to work with the entire Frobenius-unstable locus at once, it appears difficult to define this functorially, so we first define:

Definition 4.1. Given a half-integer $\ell>0$, a Frobenius-unstable vector bundle of level $\ell$ is a semi-stable vector bundle $\mathscr{F}$ of rank 2 together with a line sub-bundle of $F^{*} \mathscr{F}$ with degree equal to $\ell+\frac{p}{2} \operatorname{deg} \mathscr{F}$.

We then claim:

Proposition 4.2. $\quad$ Given a positive half-integer $\ell$, fix a line bundle $\mathscr{L}$ on $C^{(p)}$, with degree having the same parity as $2 \ell$. Then dormant torally crys-stable bundles of level $\ell$ on $C$ are equivalent to vector bundles on $C^{(p)}$ of determinant $\mathscr{L}$ which are Frobenius-unstable of level $\ell$, up to tensoring by (one of the $2^{2 g}$ ) line bundles of order 2.

Proof. First, given an $\mathscr{F}$ on $C^{(p)}$ which is semi-stable, but such that $F^{*} \mathscr{F}$ has a destabilizing sub-bundle of level $\ell$, if we denote by $\nabla^{\text {can }}$ the canonical connection associated to the Frobenius pullback (see [12, Thm. 5.1]) it is easy to see that $\left(F^{*} \mathscr{F}, \nabla^{\text {can }}\right)$ gives a dormant torally crys-stable of level $\ell$, taking into account the comment following Proposition 2.7, and noting that the fact that $r=0$ renders the additional conditions irrelevant.

Conversely, Proposition 3.3 tells us that given a torally crys-stable bundle of level $\ell$, we can choose a representing pair $(\mathscr{E}, \nabla)$ such that $\mathscr{E}$ has determinant $F^{*} \mathscr{L}$, with $\nabla$ having determinant equal to the corresponding canonical connection. Next, given the dormancy condition, the Cartier isomorphism (see [12, Thm. 5.1]) gives us an $\mathscr{F}$ on $C^{(p)}$ with determinant $\mathscr{L}$, such that $(\mathscr{E}, \nabla)=\left(F^{*} \mathscr{F}, \nabla^{\text {can }}\right)$. The destabilizing sub-bundle of $\mathscr{E}$ in the definition of level of a torally crys-stable bundle gives us the required destabilizing subbundle of $F^{*} \mathscr{F}$, but the fact that the destabilizing sub-bundle is not horizontal for $\nabla^{\text {can }}$ implies by Lemma 3.4 that $\mathscr{F}$ itself is stable. The only ambiguity in this was in the choice of $\mathscr{E}$, since once $\nabla$ is required to have a particular determinant it is uniquely determined. But $\mathscr{E}$ is determined up to tensoring by a line bundle, and for the determinant to remain unchanged, the line bundle must be of order 2. Since $p>2$, this is equivalent to tensoring $\mathscr{F}$ by a line bundle of order 2 , as desired. 
Recall that the space of semi-stable vector bundles with trivialized determinant naturally forms an algebraic stack (see $[3, \S 3, \S 8]$, where the stack is denoted $\left.\mathcal{S} \mathcal{L}_{X}(r)^{s s}\right)$. Although our results are ultimately in terms of coarse moduli spaces, it will be convenient to argue in terms of stacks.

Lemma 4.3. The stack of Frobenius-unstable vector bundles of level $\ell$ is a locally closed sub-stack of the stack of stable vector bundles (of rank 2 and the appropriate determinant).

Proof. By Lemma 3.4, Frobenius-unstable vector bundles form a substack of the stack of semi-stable vector bundles, and we see by the same lemma that they must lie within the stable locus. We thus need to check that this is a locally closed sub-stack; since both stacks deal only with quasi-coherent sheaves which are (locally) of finite presentation, and maps between such sheaves, they are clearly locally of finite presentation (i.e., on the level of rings they commute with direct limits; see [16, Prop. 4.15]), so it is easy to see that we may restrict to schemes $T$ of finite type over $S$, and in particular schemes which are Noetherian. Now, suppose we are given a vector bundle $\mathscr{F}$ of rank 2 on $C^{(p)} \times_{S} T$; we want to show that the locus of $T$ on which $F^{*} \mathscr{F}$ has a line sub-bundle, or equivalently, locally free quotient of rank 1 , of the required degree, is given functorially as a locally closed subscheme of $T$. Let $Q / T$ be the Quot scheme of quotients of $F^{*} \mathscr{F}$ with the appropriate Hilbert polynomial, and $Q^{\prime} \subset Q$ the open subscheme parametrizing locally free quotients, which then have the desired rank and degree; see [7, Thm. 2.2.4, Lem. 2.1.7, Lem. 2.1.8], and note that $Q$ is proper over $T$. We want to show that $Q^{\prime} \rightarrow T$ is an immersion. We already observed that it is a monomorphism, so by [18, Cor. I.2.13, p. 102] it suffices to check the valuative criterion for radimmersions, [18, Thm. I.2.12, p. 101] (where we note that in the statement of $\left(^{*}\right)$ in loc. cit., the phrases "horizontal morphisms on the left" and "horizontal morphisms on the right" should read "vertical morphisms on the left" and "vertical morphisms on the right"). Because $Q$ is proper over $T$, we immediately see that it is enough to show that any $k$-valued point of $Q$ whose image in $T$ is in the image of $Q^{\prime}$ must itself be in $Q^{\prime}$. Recalling that $k$ is a field, this is equivalent to the assertion that over a field, if $F^{*} \mathscr{F}$ has a locally free quotient of rank 1 and the required degree, then it has no other quotients that yield points of $Q$. Finally, we can conclude the desired statement because we are on a smooth curve, so taking a saturation of the kernel of such a quotient would produce a destabilizing line sub-bundle, and we could then apply Lemma 3.4 once more.

It thus follows via Theorem 2.11 and general moduli space theory that we 
can conclude:

Theorem 4.4. Suppose that $p>2 g-2, S=\operatorname{Spec} k$ with $k$ an algebraically closed field, and $C$ is general. Then the locus of Frobenius-unstable vector bundles of trivial determinant in the coarse moduli space $M_{2}\left(C^{(p)}\right)$ of semi-stable vector bundles of rank two and trivial determinant on $C^{(p)}$ is nonempty of dimension $2 g-4$. The locus of Frobenius-unstable vector bundles inside the coarse moduli space of semi-stable vector bundles of rank two and fixed odd determinant is non-empty of dimension $2 g-3$. More generally, the locus of Frobenius-unstable vector bundles of level $\ell$ and fixed determinant has dimension $2 g-2-2 \ell$.

Proof. Since the Frobenius-unstable locus in the moduli space is clearly the image of the disjoint union of the loci for each level, the first two assertions follow from the last. Noting that the argument of Proposition 4.2 used no hypotheses on the base to go from a Frobenius-unstable bundle to a dormant torally crys-stable bundle, we get a morphism from the stack of Frobeniusunstable bundles of level $\ell$ to the algebraic space of dormant torally crys-stable bundles of level $\ell$.

We wish to see that the locus of Frobenius-unstable bundles of level $\ell$ in $M_{2}\left(C^{(p)}\right)$ represents the étale sheafification of the stack of Frobenius-unstable bundles of level $\ell$. This is certainly well-known to the experts, but due to lack of an appropriate reference, we include a (somewhat circuitous) argument based on available citations. We first argue that the stable locus of $M_{2}\left(C^{(p)}\right)$ itself represents the sheafification of the corresponding stack: by [13, Prop. 9.1], the stable locus is the $G C$ quotient of the stack, which is determined by a universal property for maps to algebraic spaces. On the other hand, [1, Thm. 5.1.5] asserts that we can, in effect, mod out by the automorphisms of the stack of stable vector bundles to obtain a quotient stack without automorphisms, and having the same "moduli space" (i.e., algebraic space representing the sheafification). But since this quotient is already automorphism-free, by [16, Cor. 8.1.1] it is an algebraic space, and hence is itself the sheafification of the stack of stable bundles. But by construction, this quotient satisfies a universal property (in fact for maps to arbitrary stacks), so it must agree with the $G C$ quotient, and in fact be the scheme obtained by GIT, as desired. Moreover, we see from this that the stack of stable bundles is a $\mu_{2}$-gerbe over the coarse moduli space, so to conclude that the locus of Frobenius-unstable bundles represents the étale sheafification of the stack, by the previous lemma it suffices to argue that for a $\mu_{2}$-gerbe, sheafification commutes with passing to a locally closed substack. 
But this follows from [17, Prop. 2.2.1.6], which implies ideal sheaves on the gerbe are equipped with the trivial $\mu_{2}$ action, and hence correspond to ideal sheaves on the base via pushforward.

Since we have seen that the locus of Frobenius-unstable bundles of level $\ell$ in $M_{2}\left(C^{(p)}\right)$ represents the étale sheafification of the corresponding stack, any map from this stack to an algebraic space factors through the locus in question. Since the stack of dormant torally indigenous bundles is an algebraic space (see [18, Thm. I.2.7, p. 99, Prop. I.3.3, p. 104]; dormancy simply gives a further closed condition), we obtain a map from the locus of Frobenius-unstable bundles of level $\ell$ in $M_{2}\left(C^{(p)}\right)$ to the space of dormant torally indigenous bundles of level $\ell$. Furthermore, we know that this map is bijective on $k$-valued points, so we conclude the dimension of the locus of Frobenius-unstable bundles of level $\ell$ from Theorem 2.11. We may finish the proof by considering the minimal cases $\ell=\frac{1}{2}, 1$ as appropriate, as long as we know that the spaces in question are non-empty. By the flatness assertion of Theorem 2.11, it suffices to show that the locus of torally crys-stable bundles of level $\ell$ is non-empty for all $\ell>0$ on any single curve $C_{0}$, which is Lemma 3.6.

Remark 4.5. Because our lexicon was developed over arbitrary strictly Henselian bases, it is easy to check that in fact the map from the locus of Frobenius-unstable bundles to the algebraic space of dormant torally crys-stable bundles is étale; the former is therefore an explicit étale cover of the latter by a scheme.

Remark 4.6. We observe that for bundles of rank 2, there is a notable distinction between the case $p=2$ and $p>2$. For $p=2$, results of Joshi, Ramanan, Xia, Yu, Laszlo and Pauly have shown [9, Thm. 1.1], [15, Prop. 6.1 2], [8, Thm. 4.6.4] that the dimension of the Frobenius-unstable locus is $2 g-4$, independent of whether the determinant has odd or even degree. However, for $p>2$, Theorem 4.4 implies that the dimension does depend on the degree. More specifically, we see that the dimension obtained for $p=2$ is the same as that obtained for $p>2$ in the even degree case. This is perhaps not surprising given that $p=2$ is the only case for which $F^{*} \mathscr{E}$ always has even degree irrespective of the degree of $\mathscr{E}$.

We also mention that in the only case for which the number of Frobeniusunstable bundles is finite, Mochizuki's results also give the number.

Theorem 4.7. The number of Frobenius-unstable vector bundles of rank 2 and trivial determinant on a general curve $C$ of genus 2 is given by 
$\frac{2\left(p^{3}-p\right)}{3}$. Further, they all have no non-trivial deformations, in the sense that for a Frobenius-unstable bundle $\mathscr{F}$, no non-trivial first-order deformation of $\mathscr{F}$ on the fixed curve $C^{(p)}$ induces the trivial deformation of $F^{*} \mathscr{F}$.

Proof. In this case, the only possible positive integral level is $\ell=1$. By Theorem 4.2, the number of Frobenius unstable bundles is $2^{2 g}=16$ times the number of dormant torally indigenous bundles on $C$. It follows from Theorem 2.10 that dormant torally indigenous bundles on a fixed general curve $C$ have no non-trivial deformations, so in particular any Frobenius-unstable bundle has no non-trivial deformations such that the pullback remains fixed. It also follows from Theorem 2.10 that to conclude the desired formula it suffices to consider the case that $C$ is totally degenerate; we choose the totally degenerate curve obtained by gluing two copies of $\mathbb{P}^{1}$ to each other three times. By Proposition 3.5 , and the fact [18, p. 206] that a torally indigenous bundle on $\mathbb{P}^{1}$ with three marked points is determined by its radii, we find that this number is the same as the number of dormant torally indigenous bundles on $\mathbb{P}^{1}$ with three marked points, which is $\frac{p^{3}-p}{24}$ by [18, Cor. V.3.7, p. 267].

Remark 4.8. One natural question which is not addressed by these techniques, due to the necessity of working level by level, is the relationship between the strata of different levels. Particularly if one wanted to conclude, for instance, that the Frobenius-unstable locus is pure of the given dimension, one would want to show that every higher-level bundle is a specialization of one with lower (but still positive) level. However, the deformation theory raised by such a question seems substantially more delicate than the constant-level case.

\section{§5. Rational Functions with Prescribed Ramification}

For the desired applications to self-maps of $\mathbb{P}^{1}$, the statements of equivalences with dormant torally indigenous bundles are slightly more complicated. We specialize to the case $g=0$, and show:

Theorem 5.1. Fix radii $\left\{\rho_{i}\right\} \subset \mathbb{Z} / p \mathbb{Z}$ for each of the $r$ marked points on $C=\mathbb{P}^{1}$, with sign mod $p$ chosen so that $0<\rho_{i}<\frac{p}{2}$. Then there is a natural map $\varphi$ from the set of self-maps of $\mathbb{P}^{1}$ ramified to order $p-2 \rho_{i}$ at the marked points and unramified elsewhere, to the set of dormant torally indigenous bundles on $\mathbb{P}^{1}$ of radii $\left\{\rho_{i}\right\}$. We have further:

(i) The map $\varphi$ is injective after passing to equivalence classes of maps related by post-composition by automorphisms of the image. 
(ii) If the marked points are general, the map $\varphi$ is surjective.

(iii) If the marked points are general, there is a bijective correspondence between self-maps of $\mathbb{P}^{1}$ as above, and self-maps of $\mathbb{P}^{1}$ for which any even number of the ramification indices $p-2 \rho_{i}$ have been replaced by $2 \rho_{i}$.

Proof. We will once again use [22], which generalizes the construction of $[18, \S I V .2 .1]$ to arbitrarily many marked points. Since we are on $\mathbb{P}^{1}$, there need not be any ambiguity in our choices of vector bundles with connection: for $r$ even, we can choose $\mathscr{E}=\mathcal{O}\left(-\frac{r}{2}+1\right) \oplus \mathcal{O}\left(\frac{r}{2}-1\right)$, and for $r$ odd, we can choose $\mathscr{E}=\mathcal{O}\left(\frac{p-r}{2}+1\right) \oplus \mathcal{O}\left(\frac{r+p}{2}-1\right)$, and then $\nabla$ will be uniquely determined as there is only one connection with vanishing $p$-curvature on the determinant line bundles $\mathcal{O}$ and $\mathcal{O}(p)$. We then apply [22, Thm. 1.1] to conclude the existence of $\varphi$ as well as assertions (i) and (ii). Assertion (iii) is in fact unrelated to indigenous bundles; this is simply [23, Lem. 5.2], which is an essentially elementary lemma on rational functions in positive characteristic.

One conclusion is:

Corollary 5.2. $\quad$ The number of self-maps of $\mathbb{P}^{1}$ ramified to order less than $p$ at $r$ general marked points $P_{i}$ and unramified elsewhere, counted modulo automorphism of the image $\mathbb{P}^{1}$, is $2^{r-1}$ times the number of dormant torally indigenous bundles on $\mathbb{P}^{1}$ with the same marked points.

Proof. In light of the previous theorem, it suffices to check that $2^{r-1}$ gives the number of ways of replacing an even number of the $p-2 \rho_{i}$ by $2 \rho_{i}$, which is to say, the number of even subsets of $\{1, \ldots, r\}$. But this is simple enough: for instance, one sees by expanding $(1-1)^{r}$ with the binomial theorem that the number of even subsets is the same as the number of odd ones.

More importantly, as an immediate corollary of (i) of Theorem 5.1 together with the finiteness assertion of Theorem 2.10, we can conclude:

Theorem 5.3. $\quad$ Fix distinct points $P_{1}, \ldots, P_{r}$ on $\mathbb{P}^{1}$, and odd integers $e_{1}, \ldots, e_{r}$ less than $p$. Then the number of maps from $\mathbb{P}^{1}$ to itself which are ramified to order $e_{i}$ at the $P_{i}$ and unramified elsewhere, when counted modulo automorphisms of the image $\mathbb{P}^{1}$, is finite.

Remark 5.4. The parity hypothesis on the $e_{i}$ in this theorem is not expected to be necessary; indeed, if based on the desired parity of ramification 
indices one varies the determinant of the vector bundle with connection corresponding to the given torally indigenous bundle, as in [18, p. 206], one should obtain the injectivity of Theorem 5.1 (i) without parity hypotheses. However, because the preceding theorem is stated for (and only of interest for) arbitrary distinct marked points, rather than just general points, we cannot simply apply Theorem 5.1 (iii) to remove the parity hypothesis, as we did in Corollary 5.2.

In contrast, the hypothesis that all $e_{i}$ are less than $p$ is unquestionably necessary, as demonstrated by families such as $x^{p+2}+t x^{p}+x$. We give a direct proof of this finiteness result when the $P_{i}$ are general (and without assumptions on the parity or size of the $e_{i}$, as long as $\left.p \nmid e_{i}\right)$ in [23], but a direct proof for arbitrary distinct $P_{i}$ remains elusive. The proof in the context of dormant torally indigenous bundles is carried out by first enlarging to the category of nilpotent torally indigenous bundles, and proving a finiteness result there; it is this construction which is lacking in the context of self-maps of $\mathbb{P}^{1}$.

This finiteness result leads to strong non-existence statements for certain tame branched covers of $\mathbb{P}^{1}$ by $\mathbb{P}^{1}$; see $[21]$.

We conclude with a discussion of the relevance of dormant torally indigenous bundles to counting self-maps of $\mathbb{P}^{1}$ with prescribed ramification. Specifically, Proposition 3.5 allows us to obtain an alternate proof of the main theorem of $[23]$.

Theorem 5.5. Given positive integers $n \geq 3, d$ and $e_{1}, \ldots, e_{n}$ with $e_{i}<$ $p$ and $e_{i} \leq d$ for all $i$ and $2 d-2=\sum_{i}\left(e_{i}-1\right)$, the number $N_{\text {gen }}\left(\left\{e_{i}\right\}_{i}\right)$ of separable self-maps of $\mathbb{P}^{1}$ of degree $d$ and ramified to order $e_{i}$ at general $P_{i}$, modulo automorphisms of the image space, is given by

$$
\begin{gathered}
N_{\text {gen }}\left(e_{1}, e_{2}, e_{3}\right)= \begin{cases}1 & p>d \\
0 & \text { otherwise }\end{cases} \\
N_{\text {gen }}\left(\left\{e_{i}\right\}_{i}\right)=\sum_{d-e_{n-1}+1} \leq d^{\prime} \leq_{p+d-e_{n-1}-e_{n}} \begin{array}{c}
N_{\text {gen }}\left(\left\{e_{i}\right\}_{i \leq n-2}, e\right), \\
d-e_{n}+1 \\
\text { with } e=2 d^{\prime}-2 d+e_{n-1}+e_{n}-1
\end{array}
\end{gathered}
$$

Equivalently, for $n>3, N_{\text {gen }}\left(\left\{e_{i}\right\}_{i}\right)$ is given as the number of $(n-3)$ tuples of positive integers $e_{2}^{\prime}, \ldots, e_{n-2}^{\prime}$ such that any consecutive triple $e, e^{\prime}, e^{\prime \prime}$ of the sequence $e_{1}, e_{2}, e_{2}^{\prime}, e_{3}, e_{3}^{\prime}, \ldots, e_{n-2}, e_{n-2}^{\prime}, e_{n-1}, e_{n}$, with $e=e_{1}$ or some $e_{i}^{\prime}$, satisfies the following properties: 
(i) The sum $e+e^{\prime}+e^{\prime \prime}$ is odd, and less than $2 p$;

(ii) The triple $e, e^{\prime}, e^{\prime \prime}$ satisfies the triangle inequality: i.e., $e \leq e^{\prime}+e^{\prime \prime}, e^{\prime} \leq$ $e+e^{\prime \prime}$, and $e^{\prime \prime} \leq e+e^{\prime}$.

Proof. The two statements are easily seen to be equivalent by induction on $n$. The $n=3$ case is simplest; see, for instance, [23, Cor. 4.3], or [18, Intro., Thm. 1.3]. One checks by direct calculation (see [23, Cor. 5.3]) that the asserted formulas are invariant under replacing pairs of $e_{i}$ by $p-e_{i}$, so that by Theorem 5.1 (iii), it suffices to handle the case where all $e_{i}$ are odd. By Theorem 5.1, we see that $N_{\text {gen }}\left(\left\{e_{i}\right\}_{i}\right)$ then counts the number of dormant torally indigenous bundles of radii $\left\{\frac{p-e_{i}}{2}\right\}_{i}$. By Theorem 2.10, it may then be computed on a totally degenerate curve; we choose a chain with $P_{1}, P_{2}$ lying on the first component, $P_{3}$ on the second, and so forth, until $P_{n-1}$ and $P_{n}$ lie on the last component. By Proposition 3.5, and then another application of Theorem 5.1, this may then be described precisely as claimed in our second formulation, noting that the parity condition forces all the $e_{i}^{\prime}$ to also be odd, so that the sign ambiguity which produces the factor of $2^{r-1}$ in Corollary 5.2 does not arise.

Remark 5.6. Although this proof is far more circuitous in translating to vector bundles with connection, invoking Mochizuki's theory, and then translating back to maps, both chronologically and in spirit it preceded the ultimately self-contained proof of [23]. Indeed, although a self-contained argument was the original intent, after some study of the situation there remained the stumbling block of controlling degeneration from separable to inseparable maps, ultimately addressed by [23, Thm. 6.1]. However, we know from [22, Thm. 6.7] that for the special configurations of marked points where the map $\varphi$ of Theorem 5.1 fails to be surjective, the dormant torally indigenous bundles which are not in the image in fact correspond to families of self-maps of $\mathbb{P}^{1}$ with the prescribed ramification at the marked points, together with an additional ramification point of order greater than $p$ at an additional point. By [23, Prop. 5.4] such families are known to exist only for special configurations (and indeed, this is how the surjectivity of $\varphi$ is shown for general configurations of marked points). On the other hand, separable maps can certainly degenerate to inseparable maps as the ramification points are allowed to move. Now, Mochizuki's finite flatness theorem implies that dormant torally indigenous bundles can neither appear nor disappear under specialization, so the natural conclusion is that separable maps can degenerate to inseparable maps only when the ramification points move into special configurations allowing the existence of families of 
maps with exactly one ramification index greater than $p$. With this realization, it was then possible to write down examples of degenerations of connections which explicitly demonstrated the phenomenon, and finally to discover the construction which led to a self-contained proof of this statement [23, Thm. 6.1] and hence the formula for self-maps of $\mathbb{P}^{1}$ with prescribed ramification.

\section{Acknowledgements}

I would like to thank Shinichi Mochizuki for bringing the relevant results of [18] to my attention, and assisting me greatly in translating them into more familiar language. I would also like to thank Max Lieblich for his helpful conversations. Finally, I am grateful for the referee's thorough readings and many suggestions.

\section{References}

[1] D. Abramovich, A. Corti and A. Vistoli, Twisted bundles and admissible covers, Comm. Algebra 31 (2003), no. 8, 3547-3618.

[2] M. Artin, Algebraic approximation of structures over complete local rings, Inst. Hautes Études Sci. Publ. Math. No. 36 (1969), 23-58.

[3] A. Beauville and Y. Laszlo, Conformal blocks and generalized theta functions, Comm. Math. Phys. 164 (1994), no. 2, 385-419.

[4] P. Berthelot and A. Ogus, Notes on crystalline cohomology, Princeton Univ. Press, Princeton, N.J., 1978.

[5] J.-B. Bost, Algebraic leaves of algebraic foliations over number fields, Publ. Math. Inst. Hautes Études Sci. No. 93 (2001), 161-221.

[6] A. Grothendieck, Le groupe de Brauer, III: Exemples et compléments, Dix exposès sur la cohomologie des schèmas, Masson \& Cie, 1968, pp. 88-188.

[7] D. Huybrechts and M. Lehn, The geometry of moduli spaces of sheaves, Vieweg, Braunschweig, 1997.

[8] K. Joshi, S. Ramanan, E. Z. Xia and J.-K. Yu, On vector bundles destabilized by Frobenius pull-back, Compos. Math. 142 (2006), no. 3, 616-630.

[9] K. Joshi and E. Z. Xia, Moduli of vector bundles on curves in positive characteristics, Compositio Math. 122 (2000), no. 3, 315-321.

[10] F. Kato, Log smooth deformation theory, Tohoku Math. J. (2) 48 (1996), no. 3, 317-354.

[11] K. Kato, Logarithmic structures of Fontaine-Illusie, in Algebraic analysis, geometry, and number theory (Baltimore, MD, 1988), 191-224, Johns Hopkins Univ. Press, Baltimore, MD.

[12] N. M. Katz, Nilpotent connections and the monodromy theorem: Applications of a result of Turrittin, Inst. Hautes Études Sci. Publ. Math. No. 39 (1970), 175-232.

[13] S. Keel and S. Mori, Quotients by groupoids, Ann. of Math. (2) 145 (1997), no. 1, 193-213.

[14] H. Lange and C. Pauly, On Frobenius-destabilized rank-2 vector bundles over curves, Comm. Math. Helv., to appear.

[15] Y. Laszlo and C. Pauly, The action of the Frobenius maps on rank 2 vector bundles in characteristic 2, J. Algebraic Geom. 11 (2002), no. 2, 219-243.

[16] G. Laumon and L. Moret-Bailly, Champs algébriques, Springer, Berlin, 2000.

[17] M. Lieblich, Moduli of twisted sheaves, Duke Math. J., to appear. 
[18] S. Mochizuki, Foundations of p-adic Teichmüller theory, Amer. Math. Soc., Providence, RI, 1999.

[19] A. Ogus, F-crystals, Griffiths transversality, and the Hodge decomposition, Astérisque No. 221 (1994), ii+183 pp.

[20] B. Osserman, Frobenius-unstable bundles and p-curvature, Transactions of the AMS, to appear.

[21] , Linear series and existence of branched covers, preprint.

$[22]-$ Logarithmic connections with vanishing p-curvature, preprint.

[23] $\_$, Rational functions with given ramification in characteristic $p$, Compositio Mathematica, 142 (2006), no. 2, 433-450.

[24] A. Grothendieck with J. Dieudonné, Éléments de géométrie algébrique: III. Étude cohomologique des faisceaux cohérents, premiére partie, No. 11, Publications mathématiques de l'I.H.É.S., no. 2, Institut des Hautes Études Scientifiques, 1961.

[25] Éléments de géométrie algébrique: IV. Étude locale des schémas et des morphismes de schémas, troisiéme partie, No. 28, Publications mathématiques de l'I.H.É.S., no. 2, Institut des Hautes Études Scientifiques, 1966. 